\title{
Lattice Boltzmann Simulation of Collision between 2D Circular Particles Suspension in Couette Flow
}

\author{
Li-Zhong Huang ${ }^{1}$ and De-Ming $\mathrm{Nie}^{2}$ \\ ${ }^{1}$ Institute of Fluid Engineering, Zhejiang University, Hangzhou 310027, China \\ ${ }^{2}$ College of Metrology and Technology Engineering, China Jiliang University, Hangzhou 310018, China \\ Correspondence should be addressed to De-Ming Nie; nieinhz@cjlu.edu.cn
}

Received 23 October 2013; Accepted 5 November 2013

Academic Editor: Jianzhong Lin

Copyright (C) 2013 L.-Z. Huang and D.-M. Nie. This is an open access article distributed under the Creative Commons Attribution License, which permits unrestricted use, distribution, and reproduction in any medium, provided the original work is properly cited.

Collision between 2D circular particles suspension in Couette flow is simulated by using multiple-relaxation-time based lattice Boltzmann and direct forcing/fictitious domain method in this paper. The patterns of particle collisions are simulated and analyzed in detail by changing the velocity of top and bottom walls in the Couette flow. It can be seen from the simulation results that, while the velocity is large enough, the number of collisions between particles will change little as this velocity varies.

\section{Introduction}

Multiphase flow is a very important branch of fluid mechanics, while fluid-solid two-phase flow is the main part of such flow. And fluid-solid two-phase flow is very common in nature and industry, such as raindrop formation, material science, chemical industry, aerosol deposition, fluidized beds, and injection molding machine $[1,2]$. Particle collisions, which will influence the performance of product, happen occasionally in those processes. So it is very meaningful to do some research to understand particle collisions and then take control to improve the performance of product.

Even with the help of the precise and advanced particle image velocity (PIV) instrument, it is very difficult to observe the phenomenon of particle collisions in detail. Comparing the experimental measurement, numerical simulation has great advantages to investigate particle collisions in fluid-solid two-phase flow, especially for direct numerical simulation (DNS) methods. The lattice Boltzmann method (LBM), one of the best DNS methods with several remarkable advantages, was first proposed by Ladd [3] and then improved by Aidun et al. [4] to simulate particles suspended in a viscous fluid. Feng and Michaelides [5] and Tian et al. [6] united immersed boundary method (IBM) into LBM to deal with fluid-solid interface problem. In the past several decades the LBM method was proved robust and efficient for particulate flows, especially in the case of large number of particles [712]. Nie and Lin [13] developed a single-relaxation-time (SRT) based lattice Boltzmann-direct forcing/fictitious domain (SRT LB-DF/FD) method to simulate particle suspensions and then improved to multiple-relaxation-time (MRT LB$\mathrm{DF} / \mathrm{FD})$.

Several papers investigated particle collisions in threedimensional homogeneous isotropic turbulence [14-18] but not by using DNS method. In this paper, DNS method is adopted to simulate particle collisions. Because the MRT model has better ability of computing pressure and more time saving than the SRT model, the MRT LB DF/FD method is utilized. Firstly, this method is introduced in detail in Section 2. Secondly, simulation problem is described in Section 3. And finally, several simulation results and conclusions are presented in Section 4.

\section{Numerical Method}

2.1. The MRT LB DF/FD Method. The lattice Boltzmann method based on the multiple-relaxation-time (MRT) collision model is adopted in this paper [13]. The discrete 
equations can be written as follows:

$$
f_{i}\left(\mathbf{x}+\mathbf{e}_{i} \Delta t, t+\Delta t\right)=f_{i}(\mathbf{x}, t)-M^{-1} S\left[\mathbf{m}-\mathbf{m}^{(\mathrm{eq})}\right]+\mathbf{F} \Delta t,
$$

where $S$ is the diagonal collision matrix, $S=\operatorname{diag}\left(0, s_{e}, s_{\mathcal{\varepsilon}}, 0\right.$, $s_{q}, 0, s_{q}, s_{v}, s_{v}$ ), and $M$ is the transform matrix,

$$
M=\left[\begin{array}{ccccccccc}
1 & 1 & 1 & 1 & 1 & 1 & 1 & 1 & 1 \\
-4 & -1 & -1 & -1 & -1 & 2 & 2 & 2 & 2 \\
4 & -2 & -2 & -2 & -2 & 1 & 1 & 1 & 1 \\
0 & 1 & 0 & -1 & 0 & 1 & -1 & -1 & 1 \\
0 & -2 & 0 & 2 & 0 & 1 & -1 & -1 & 1 \\
0 & 0 & 1 & 0 & -1 & 1 & 1 & -1 & 1 \\
0 & 0 & -2 & 0 & 2 & 1 & 1 & -1 & -1 \\
0 & 1 & -1 & 1 & -1 & 0 & 0 & 0 & 0 \\
0 & 0 & 0 & 0 & 0 & 1 & -1 & 1 & -1
\end{array}\right]
$$

The moment space spanned by $\mathbf{m}_{i}$ and the velocity space spanned by $f_{i}$ are related by a linear mapping, $\mathbf{m}=M f$; that is, $f=M^{-1} \mathbf{m}$. The D2Q9 model is used in 2D simulation, and the discrete velocities are listed as the follows:

$$
\mathbf{c}_{i}= \begin{cases}(0,0) & i=0, \\ ( \pm 1,0) c,(0, \pm 1) c & i=1-4 \\ ( \pm 1, \pm 1) c,( \pm 1, \pm 1) c & i=5-8\end{cases}
$$

where $c=\Delta x / \Delta t$ is the lattice speed, among which $\Delta x$ is the lattice spacing, and $\Delta t$ is the time step. For D2Q9 model, the corresponding nine moments are given by [19]

$$
\mathbf{m}=\left(\rho, e, \varepsilon, j_{x}, q_{x}, j_{y}, q_{y}, p_{x x}, p_{x y}\right)^{\dagger},
$$

where $\rho$ is the mean density and $j_{x}=\rho u_{x}, j_{y}=\rho u_{y}$ are the conserved moments. Moreover, the other nonconserved moments are listed as follows:

$$
\begin{gathered}
e^{(\mathrm{eq})}=-2 \rho+\frac{3 \mathbf{j} \cdot \mathbf{j}}{\rho}, \quad \varepsilon^{(\mathrm{eq})}=\rho-\frac{3 \mathbf{j} \cdot \mathbf{j}}{\rho}, \quad q_{x}^{(\mathrm{eq})}=-j_{x}, \\
q_{y}{ }^{(\mathrm{eq})}=-j_{y}, \quad p_{x x}^{(\mathrm{eq})}=\frac{\left(j_{x}^{2}-j_{y}^{2}\right)}{\rho}, \quad p_{x y}^{(\mathrm{eq})}=\frac{j_{x} j_{y}}{\rho} .
\end{gathered}
$$

According to Guo et al. [20], the forcing term $\mathbf{F}$ is defined as follows:

$$
\begin{gathered}
\mathbf{F}=M^{-1}\left(I-\frac{1}{2} S\right) M \overline{\mathbf{F}}, \\
\overline{F_{i}}=w_{i}\left[\frac{\mathbf{c}_{i} \cdot \boldsymbol{\alpha}}{c_{s}^{2}}+\frac{\mathbf{u} \boldsymbol{\alpha}: \mathbf{c}_{i} \mathbf{c}_{i}}{c_{s}^{4}}-\frac{\mathbf{u} \boldsymbol{\alpha}: c_{s}^{2} I}{c_{s}^{4}}\right],
\end{gathered}
$$

where $\boldsymbol{\alpha}$ is the acceleration due to the external force and $w_{i}$ are weights related to the lattice model which are chosen as $w_{0}=4 / 9 ; w_{i}=1 / 9, i=1 \sim 4 ; w_{i}=1 / 36, i=5 \sim 8$. The speed of sound $c_{s}$ is equal to $c / 3^{1 / 2}$. By applying the Taylor expansion

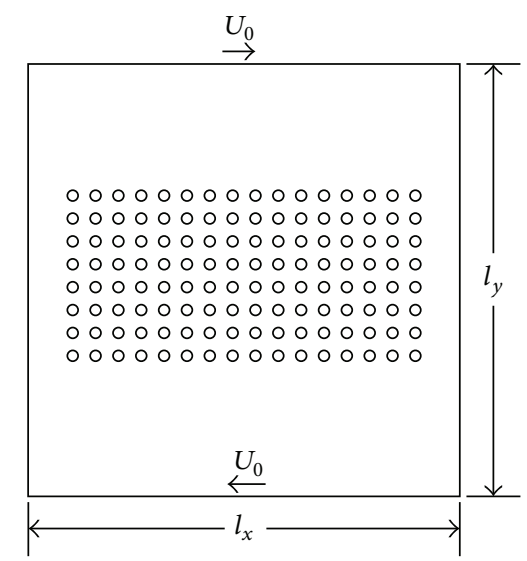

FIGURE 1: Schematic diagram of geometry.

techniques and the Chapman-Enskog analysis, (1) leads to the hydrodynamic equations that are shown as follows:

$$
\nabla \cdot \mathbf{u}=0, \quad \rho_{f}\left(\frac{\partial \mathbf{u}}{\partial t}+(\mathbf{u} \cdot \nabla) \mathbf{u}\right)=-\nabla p+\mu \nabla^{2} \mathbf{u}+\mathbf{a} .
$$

The shear viscosity and the bulk viscosity can be defined as follows [21]:

$$
\nu=\frac{1}{3}\left(\frac{1}{s_{v}}-\frac{1}{2}\right) c \Delta x, \quad \zeta=\frac{1}{3}\left(\frac{1}{s_{e}}-\frac{1}{2}\right) c \Delta x .
$$

2.2. Collisions Model. A collision model is needed to avoid particles overlapping. Then the short-range repulsive force developed by Wan and Turek [22] is utilized in this paper because it is easily carried out in the code. For particleparticle collisions, the short-range repulsive force is calculated by

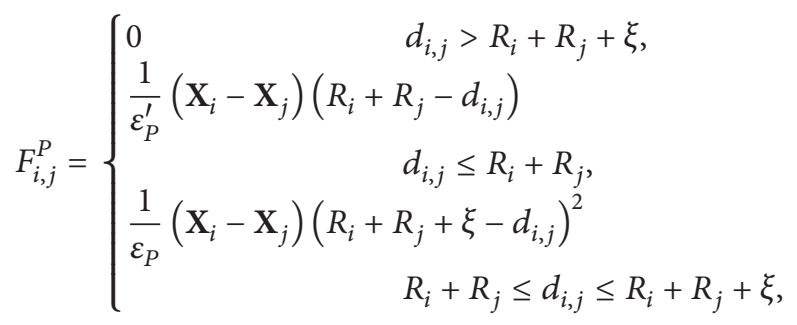

where $\mathbf{X}_{i}$ and $\mathbf{X}_{j}$ are the mass center coordinates of the $i$ th and $j$ th particle, $R_{i}$ and $R_{j}$ are their radius, $d_{i, j}$ is the distance between their mass centers which equals to $\left|\mathbf{X}_{i}-\mathbf{X}_{j}\right|$, $\xi$ is the force range which is usually set to be one or two lattice spacing, and $\varepsilon_{p}^{\prime}$ and $\varepsilon_{p}$ are two small positive stiffness parameters for particle-particle collisions. 


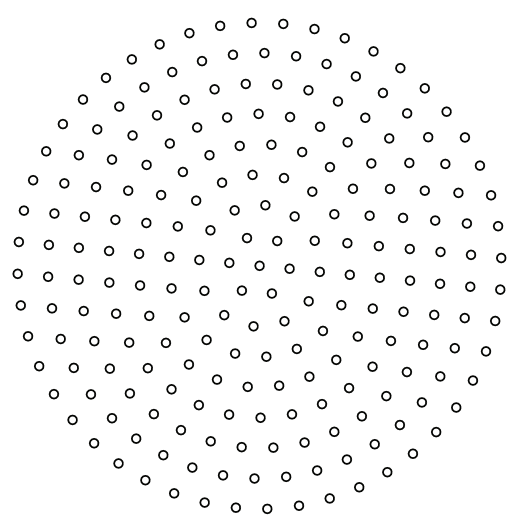

FIGURE 2: Arrangements of Lagrangian points.

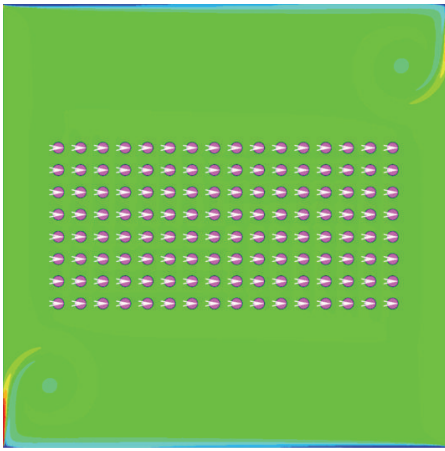

(a) $t^{\prime}=1.5$

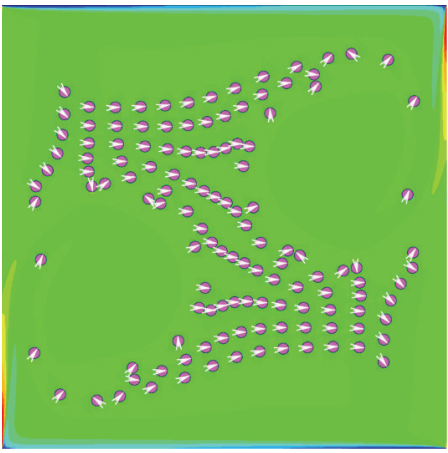

(d) $t^{\prime}=5.5$

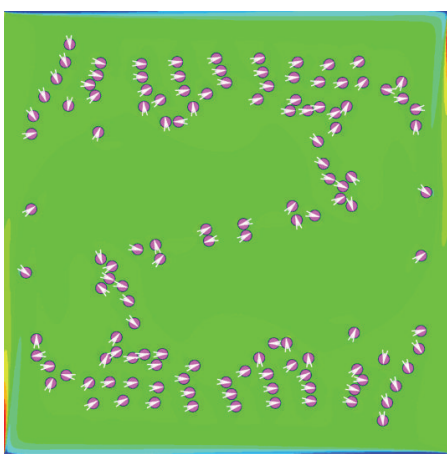

(g) $t^{\prime}=8.0$

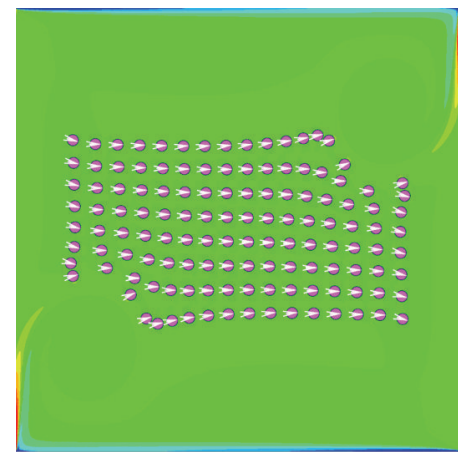

(b) $t^{\prime}=3.0$

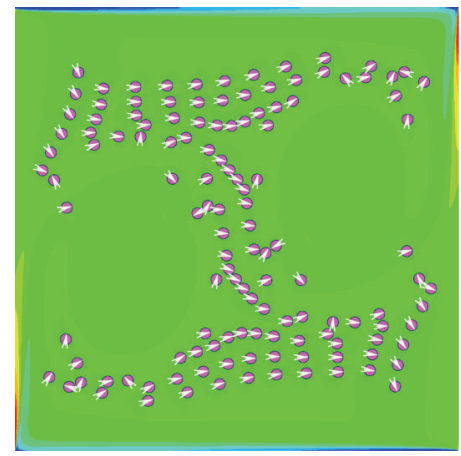

(e) $t^{\prime}=6.5$

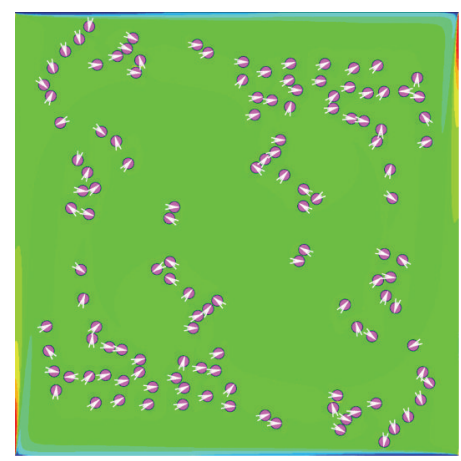

(h) $t^{\prime}=9.0$

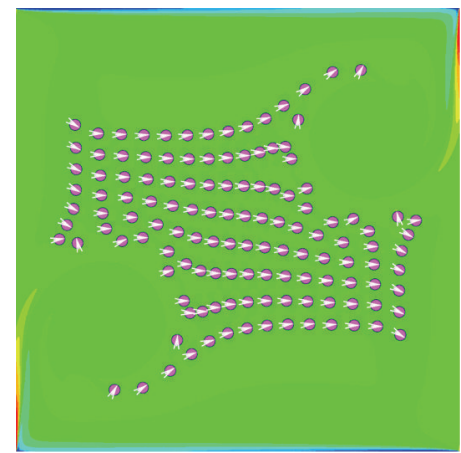

(c) $t^{\prime}=4.0$

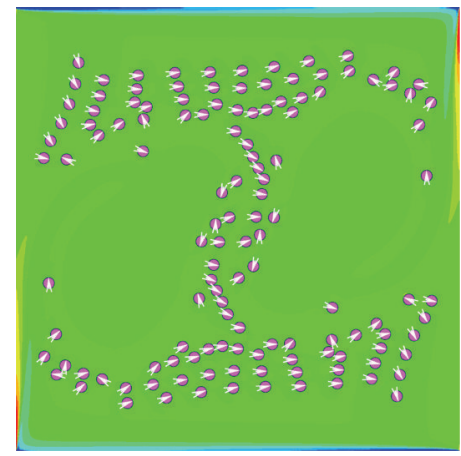

(f) $t^{\prime}=7.0$

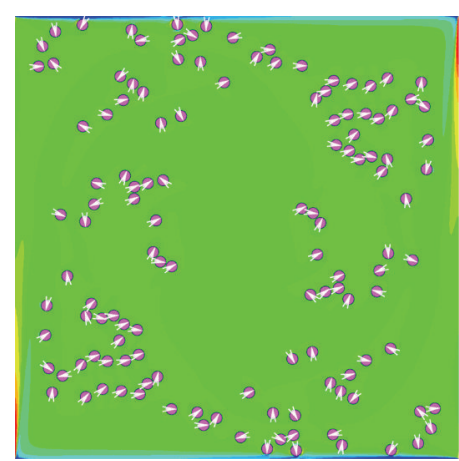

(i) $t^{\prime}=10.0$

FIgURE 3: Vorticity contours when $U_{0}=0.1 \mathrm{~m} / \mathrm{s}$. 


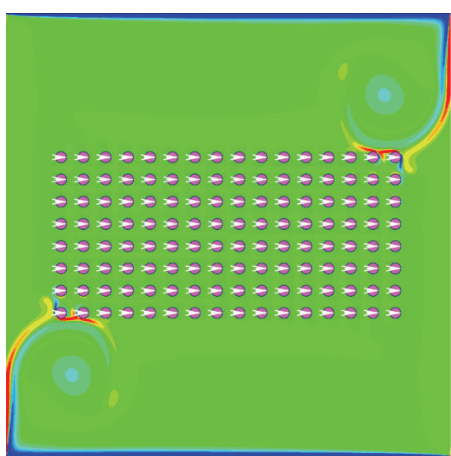

(a) $t^{\prime}=1.5$

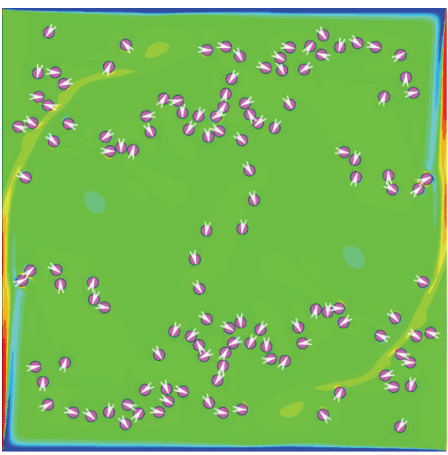

(d) $t^{\prime}=5.3$

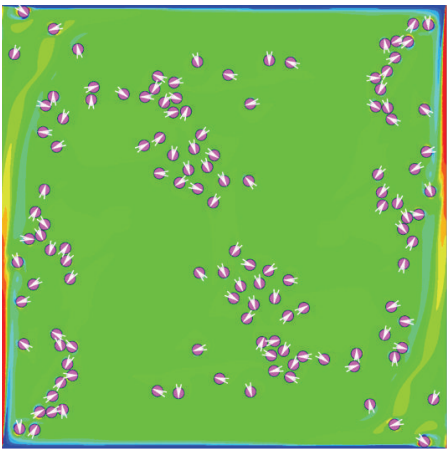

(g) $t^{\prime}=7.4$

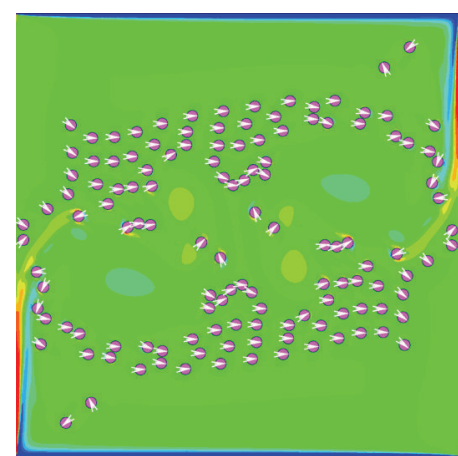

(b) $t^{\prime}=3.0$

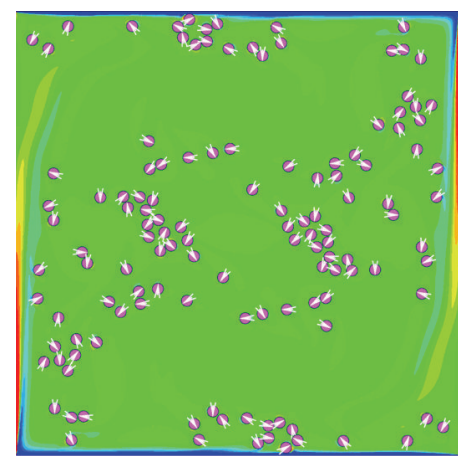

(e) $t^{\prime}=6.6$

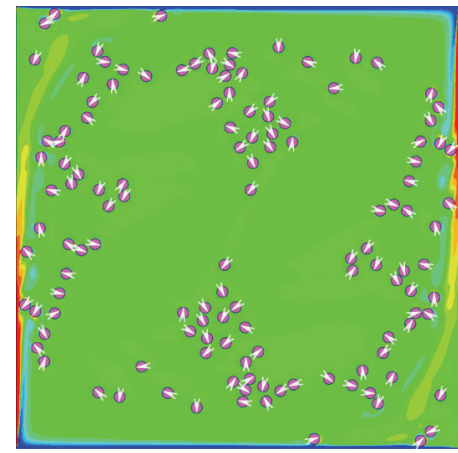

(h) $t^{\prime}=7.8$

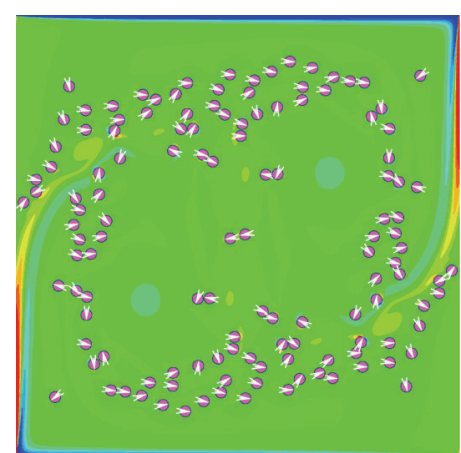

(c) $t^{\prime}=4.0$

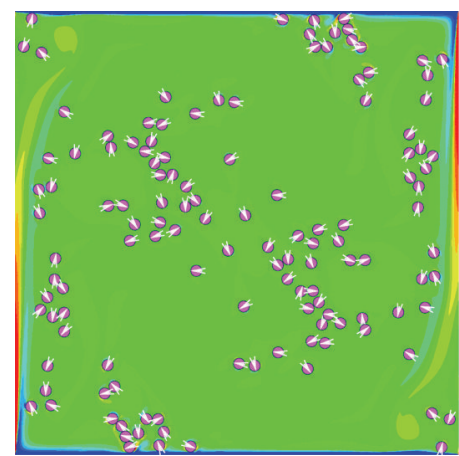

(f) $t^{\prime}=7.0$

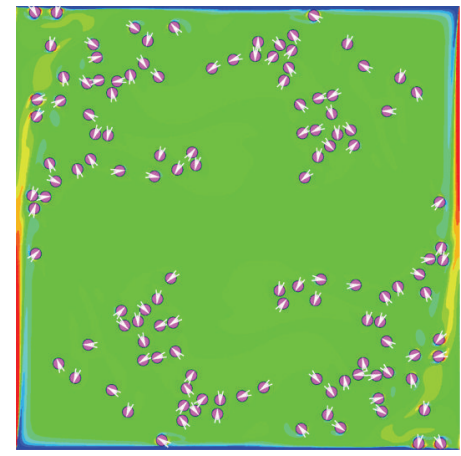

(i) $t^{\prime}=8.2$

FIGURE 4: Vorticity contours when $U_{0}=0.2 \mathrm{~m} / \mathrm{s}$.

But for particle-wall collisions, the corresponding shortrange repulsive force is determined as follows:

$$
F_{i, j}^{W}= \begin{cases}0 & d_{i}^{\prime}>2 R_{i}+\xi, \\ \frac{1}{\varepsilon_{W}^{\prime}}\left(\mathbf{X}_{i}-\mathbf{X}_{i}^{\prime}\right)\left(2 R_{i}-d_{i}^{\prime}\right) & d_{i}^{\prime} \leq 2 R_{i}, \\ \frac{1}{\varepsilon_{W}}\left(\mathbf{X}_{i}-\mathbf{X}_{i}^{\prime}\right)\left(2 R_{i}+\xi-d_{i}^{\prime}\right)^{2} & 2 R_{i} \leq d_{i}^{\prime} \leq 2 R_{i}+\xi,\end{cases}
$$

where $\mathbf{X}_{i}^{\prime}$ is the coordinate of the nearest imaginary particle located on the boundary nearby the $i$ th particle, $d_{i}^{\prime}$ is the distance between the imaginary particle and the real one which equals to $\left|\mathbf{X}_{i}-\mathbf{X}_{i}^{\prime}\right|$, and $\varepsilon_{W}^{\prime}$ and $\varepsilon_{W}$ are the other two small positive stiffness parameters for particle-wall collisions. In this paper, $\varepsilon_{p}^{\prime}, \varepsilon_{p}, \varepsilon_{W}^{\prime}$, and $\varepsilon_{W}$ are all set to be le-7.

\section{Problem Description}

The main purpose of this paper is to study collisions between 2D circular particles suspension in Couette flow. The schematic diagram of geometry is shown in Figure 1 which is constructed by four solid walls, $l x=l y=8 \mathrm{~cm}$, and mesh element is 640000. The left and right walls are fixed, while the top wall moves in the right direction with a velocity of $U_{0}$ and the bottom wall moves in the left direction at the same velocity. 128 circular particles ( 8 rows, 16 columns) are placed in the middle of this domain, and the distance of center of two neighborhood particles in horizontal and vertical direction is 


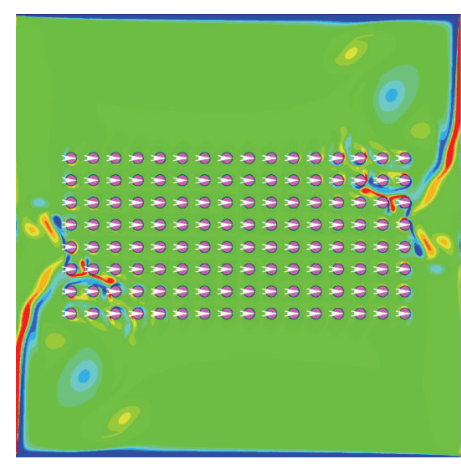

(a) $t^{\prime}=1.5$

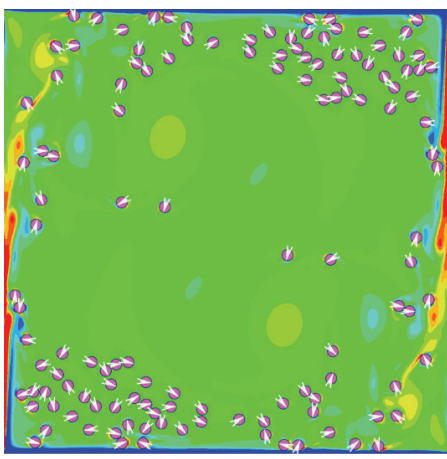

(d) $t^{\prime}=5.3$

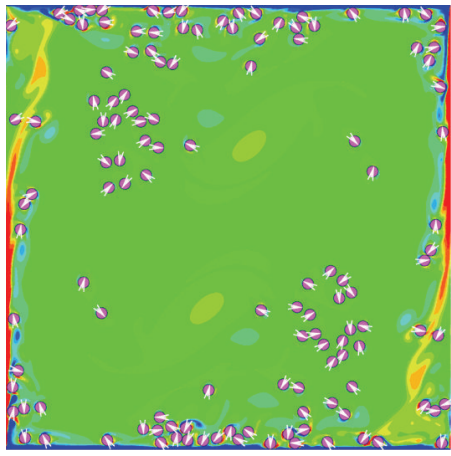

(g) $t^{\prime}=7.4$

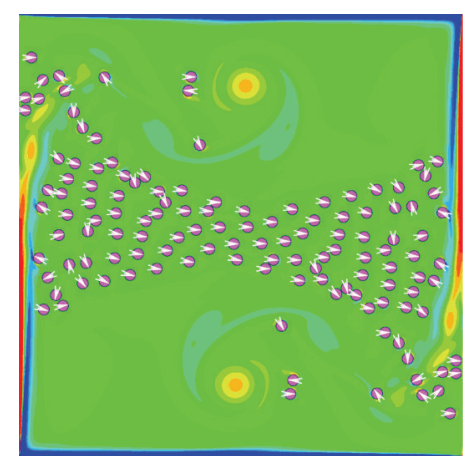

(b) $t^{\prime}=3.0$

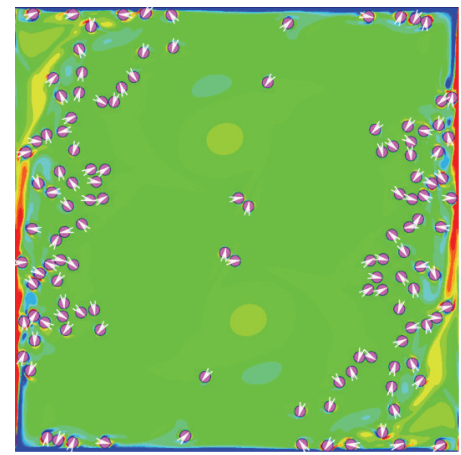

(e) $t^{\prime}=6.6$

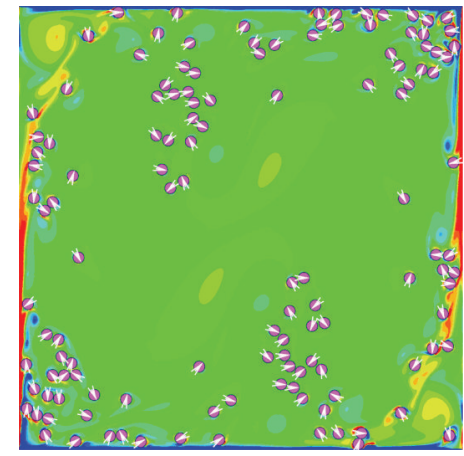

(h) $t^{\prime}=7.8$

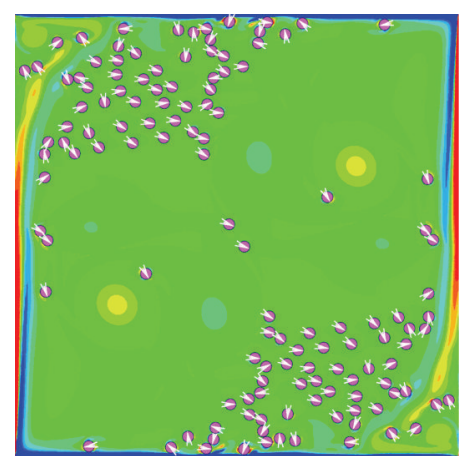

(c) $t^{\prime}=4.0$

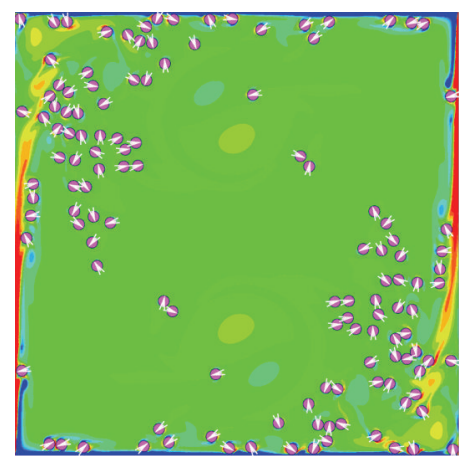

(f) $t^{\prime}=7.0$

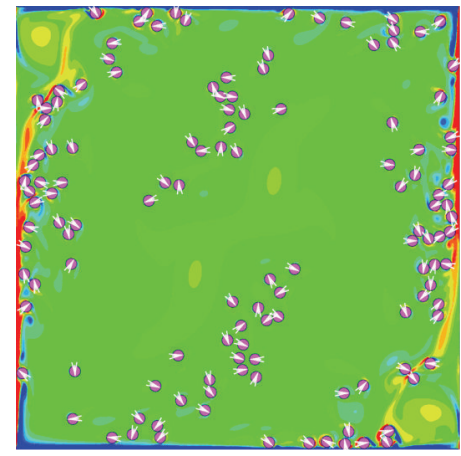

(i) $t^{\prime}=8.2$

FIGURE 5: Vorticity contours when $U_{0}=0.3 \mathrm{~m} / \mathrm{s}$.

two times of the diameter of particle. In order to ignore the influence of gravity, the density of particles is set to be same as the suspension fluid, $\rho_{p}=\rho_{f}=1.0 \mathrm{~g} / \mathrm{cm}^{3}$. The kinematic viscosity of the fluid is set to be $0.01 \mathrm{~cm}^{2} \mathrm{~s}^{-1}$. For each particle, the diameter is $0.2 \mathrm{~cm}$ and the arrangement of Lagrangian points is shown in Figure 2.

\section{Simulation Results and Conclusions}

In this paper, when $t^{\prime}=t / 10^{4} \mathrm{~s} \leq 1.5$, the circular particles are fixed to generate a fully developed Couette flow which can be seen from Figures 3(a), 4(a), and 5(a). And when $t^{\prime}>1.5$, those particles begin to move in the suspension fluid. At first, $U_{0}$ is set to be very small which equals to $0.1 \mathrm{~m} / \mathrm{s}$ and the instantaneous vorticity contours can be seen from Figures 3(a)-3(i). Obviously, the vorticity is not strong enough which makes the number of collisions very big.

Instantaneous vorticity contours for $U_{0}=0.2 \mathrm{~m} / \mathrm{s}$ at several interval times are shown in Figures 4(a)-4(i). It can be seen from those figures that the particles are moving by the influence of the two main symmetry vortices. Even when $t^{\prime}=8.2$, the vortex contour is rotational symmetry from the center of the simulation domain.

But when $U_{0}=0.3 \mathrm{~m} / \mathrm{s}$, Figures $5(\mathrm{a})-5(\mathrm{i})$ show that the circular particles are agglomerated near the four sidewalls by the influence of the two main vortices. And when $t^{\prime}>7.8$, the particles are no longer rotational symmetry from the center of the simulation domain, distributed at completely random. The vortex patterns are totally different when $U_{0}$ varies from $0.1 \mathrm{~m} / \mathrm{s}$ to $0.3 \mathrm{~m} / \mathrm{s}$; however, the number of particle collisions 


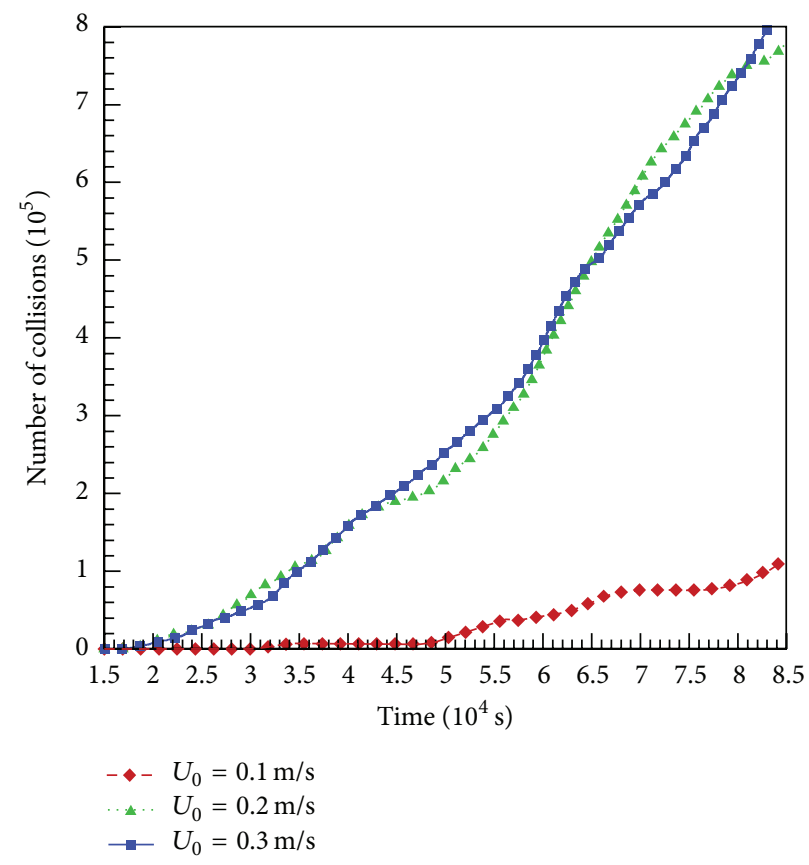

FIgure 6: Comparison of collision number with different $U_{0}$.

varies little when $U_{0}$ is large enough which can be seen from Figure 6.

\section{Acknowledgment}

This work is supported by the National Natural Science Foundation of China with Grant 11132008.

\section{References}

[1] J. Lin, X. Shi, and Z. Yu, "The motion of fibers in an evolving mixing layer," International Journal of Multiphase Flow, vol. 29, no. 8, pp. 1355-1372, 2003.

[2] L. Jianzhong, Z. Weifeng, and Y. Zhaosheng, "Numerical research on the orientation distribution of fibers immersed in laminar and turbulent pipe flows," Journal of Aerosol Science, vol. 35, no. 1, pp. 63-82, 2004.

[3] A. J. C. Ladd, "Numerical simulations of particulate suspensions via a discretized Boltzmann equation. I. Theoretical foundation," Journal of Fluid Mechanics, vol. 271, pp. 285-309, 1994.

[4] C. K. Aidun, Y. Lu, and E.-J. Ding, "Direct analysis of particulate suspensions with inertia using the discrete Boltzmann equation," Journal of Fluid Mechanics, vol. 373, pp. 287-311, 1998.

[5] Z.-G. Feng and E. E. Michaelides, "The immersed boundarylattice Boltzmann method for solving fluid-particles interaction problems," Journal of Computational Physics, vol. 195, no. 2, pp. 602-628, 2004.

[6] F.-B. Tian, H. Luo, L. Zhu, J. C. Liao, and X.-Y. Lu, "An efficient immersed boundary-lattice Boltzmann method for the hydrodynamic interaction of elastic filaments," Journal of Computational Physics, vol. 230, no. 19, pp. 7266-7283, 2011.

[7] L. Jianzhong, S. Xing, and Y. Zhenjiang, "Effects of the aspect ratio on the sedimentation of a fiber in Newtonian fluids," Journal of Aerosol Science, vol. 34, no. 7, pp. 909-921, 2003.
[8] X. Shi, J. Lin, and Z. Yu, "Discontinuous Galerkin spectral element lattice Boltzmann method on triangular element," International Journal for Numerical Methods in Fluids, vol. 42, no. 11, pp. 1249-1261, 2003.

[9] J.-Z. Lin, Y.-L. Wang, and J. A. Olsen, "Sedimentation of rigid cylindrical particles with mechanical contacts," Chinese Physics Letters, vol. 22, no. 3, pp. 628-631, 2005.

[10] L. Jianzhong and K. Xiaoke, "Fiber orientation distributions in a suspension flow through a parallel plate channel containing a cylinder," Journal of Composite Materials, vol. 43, no. 12, pp. 1373-1390, 2009.

[11] X. K. Ku and J. Z. Lin, "Inertial effects on the rotational motion of a fibre in simple shear flow between two bounding walls," Physica Scripta, vol. 80, no. 2, Article ID 025801, 2009.

[12] X. Ku and J. Lin, "Numerical simulation of the flows over two tandem cylinders by lattice Boltzmann method," Modern Physics Letters B, vol. 19, no. 28-29, pp. 1551-1554, 2005.

[13] D. Nie and J. Lin, "A LB-DF/FD method for particle suspensions," Communications in Computational Physics, vol. 7, no. 3, pp. 544-563, 2010.

[14] L. I. Zaichik, O. Simonin, and V. M. Alipchenkov, "Collision rates of bidisperse inertial particles in isotropic turbulence," Physics of Fluids, vol. 18, no. 3, Article ID 035110, 2006.

[15] L. I. Zaichik, O. Simonin, and V. M. Alipchenkov, "Two statistical models for predicting collision rates of inertial particles in homogeneous isotropic turbulence," Physics of Fluids, vol. 15, no. 10, pp. 2995-3005, 2003.

[16] L.-P. Wang, A. S. Wexler, and Y. Zhou, "On the collision rate of small particles in isotropic turbulence. I. Zero-inertia case," Physics of Fluids, vol. 10, no. 1, pp. 266-276, 1998.

[17] Y. Zhou, A. S. Wexler, and L.-P. Wang, "On the collision rate of small particles in isotropic turbulence. II. Finite inertia case," Physics of Fluids, vol. 10, no. 5, pp. 1206-1216, 1998. 
[18] C. N. Franklin, P. A. Vaillancourt, M. K. Yau, and P. Bartello, "Collision rates of cloud droplets in turbulent flow," Journal of the Atmospheric Sciences, vol. 62, no. 7, pp. 2451-2466, 2005.

[19] L.-S. Luo, W. Liao, X. Chen, Y. Peng, and W. Zhang, "Numerics of the lattice Boltzmann method: effects of collision models on the lattice Boltzmann simulations," Physical Review E, vol. 83, no. 5, Article ID 056710, 2011.

[20] Z. Guo, C. Zheng, and B. Shi, "Discrete lattice effects on the forcing term in the lattice Boltzmann method," Physical Review E, vol. 65, no. 4, Article ID 046308, 6 pages, 2002.

[21] D. d'Humières, I. Ginzburg, M. Krafczyk, P. Lallemand, and L.S. Luo, "Multiple-relaxation-time lattice Boltzmann models in three dimensions," The Royal Society of London, vol. 360, no. 1792, pp. 437-451, 2002.

[22] D. Wan and S. Turek, "An efficient multigrid-FEM method for the simulation of solid-liquid two phase flows," Journal of Computational and Applied Mathematics, vol. 203, no. 2, pp. 561-580, 2007. 


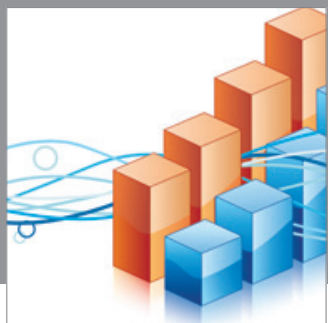

Advances in

Operations Research

mansans

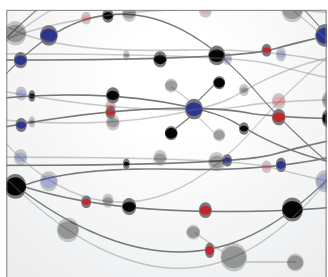

The Scientific World Journal
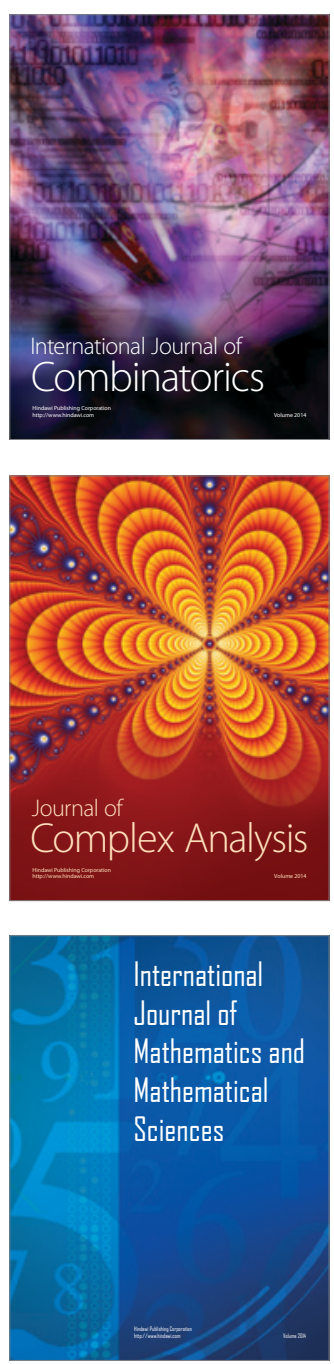
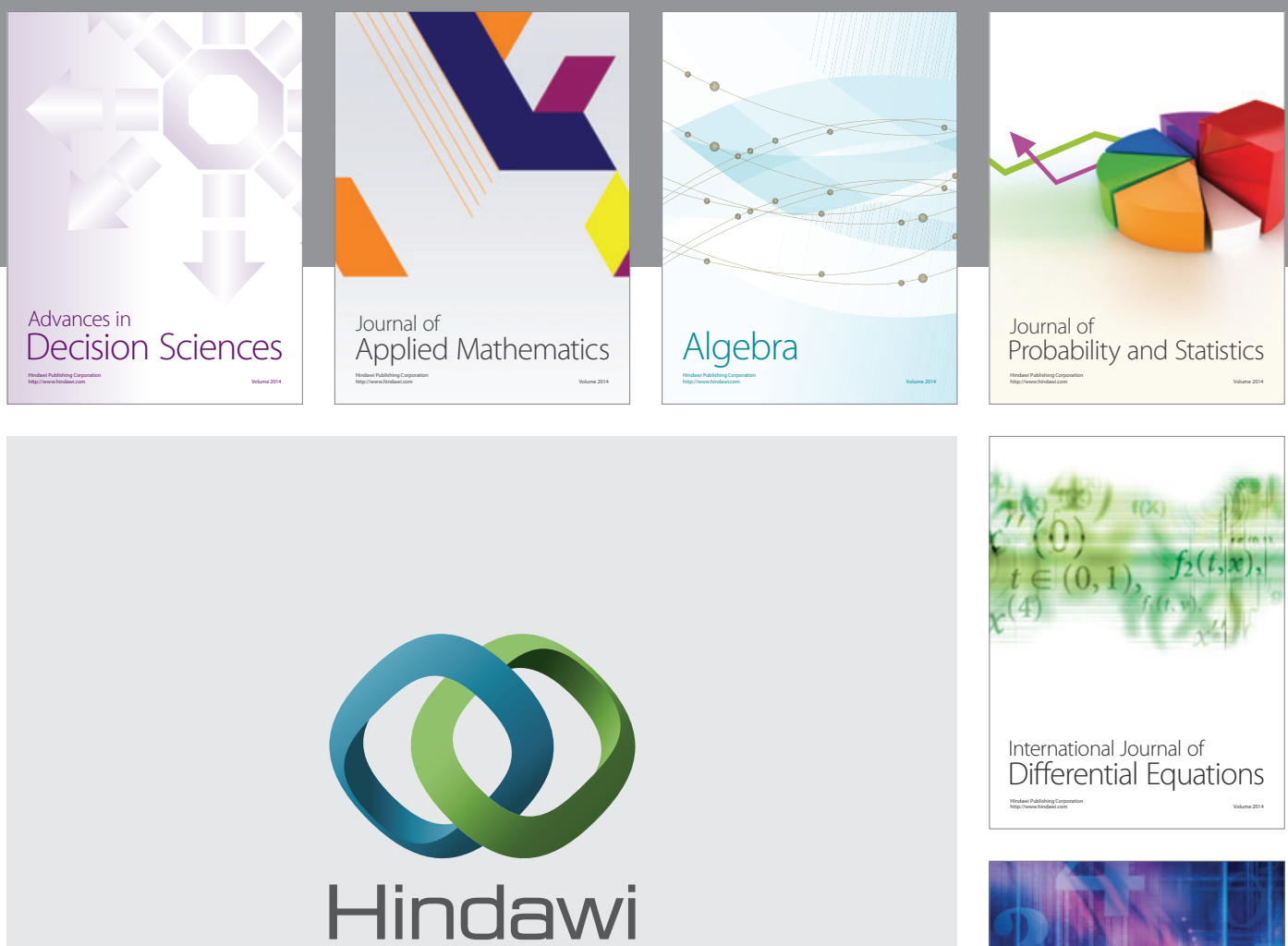

Submit your manuscripts at http://www.hindawi.com
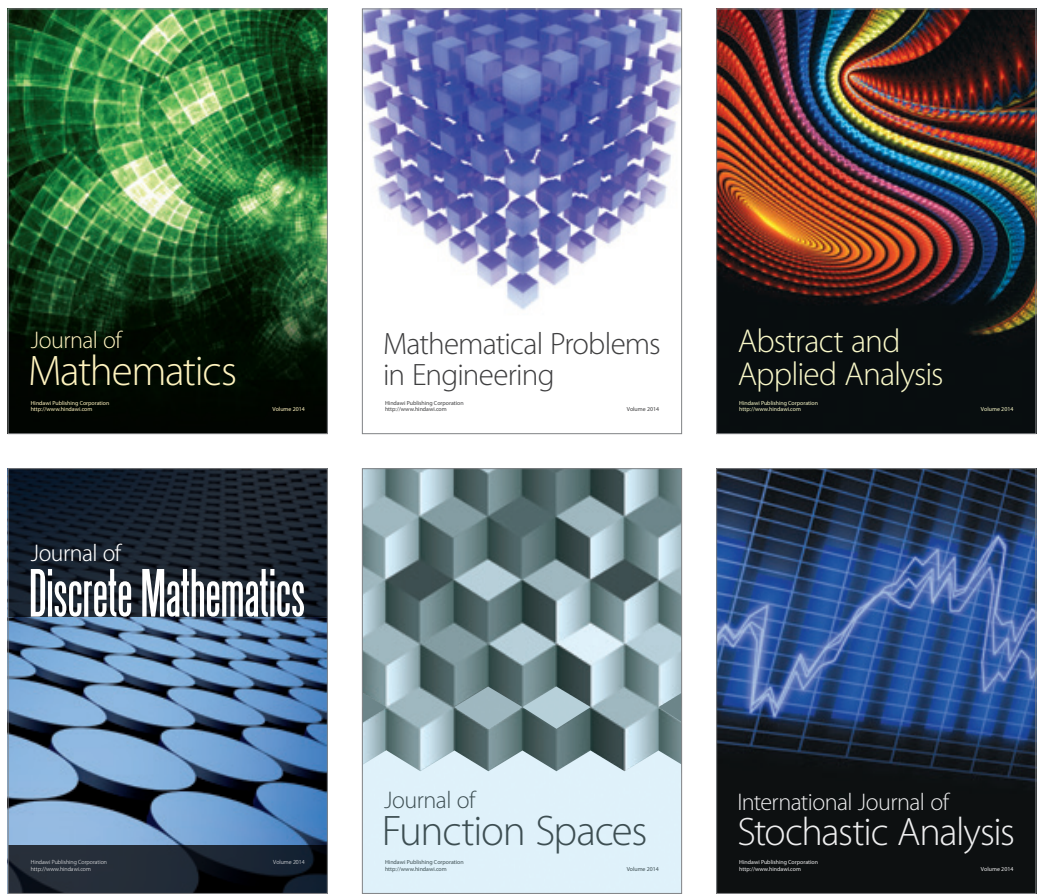

Journal of

Function Spaces

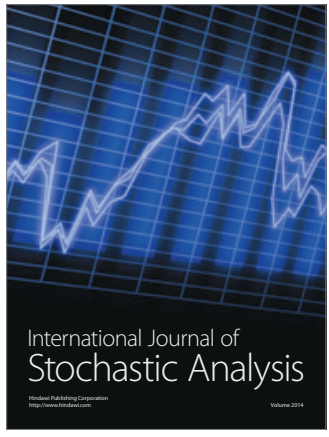

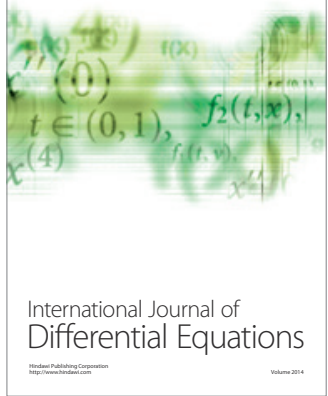
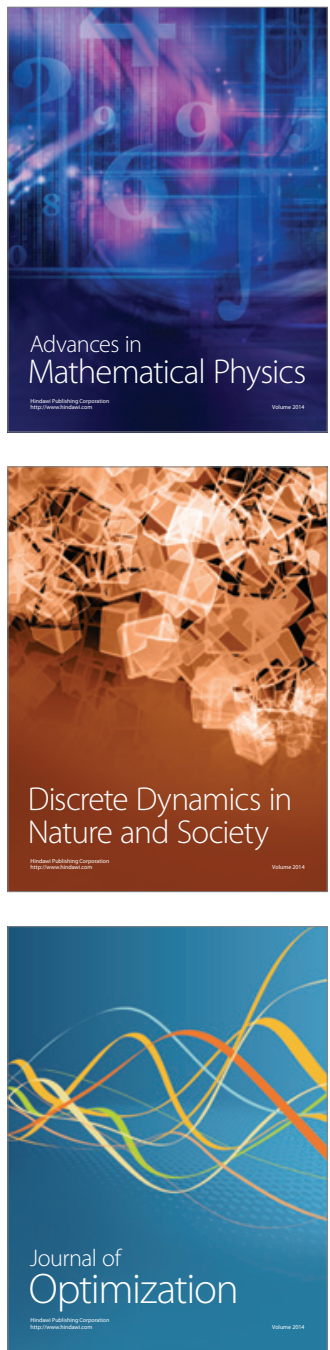